\title{
Type I strains in human toxoplasmosis: myth or reality?
}

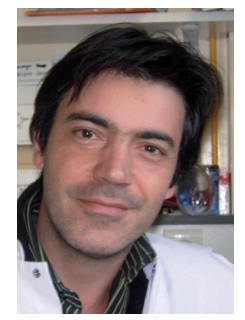

"...the importance of type I strains in toxoplasmosis

has been largely overestimated in the literature owing to a collection bias, or 'iceberg effect', and method issues."

\section{Daniel Ajzenberg}

Centre National de Référence (CNR) Toxoplasmose/Toxoplasma Biological Resource Center (BRC), Centre Hospitalier-Universitaire Dupuytren, Limoges, 87042, France and Laboratoire de Parasitologie-Mycologie, EA 3174-NETEC, Faculté de Médecine, Université de Limoges, Limoges, 87025, France m Tel.: +33 555058734 - Fax: +33 555056177 . ajz@unilim.fr

The first human strain of Toxoplasma gondii, known as RH strain, was originally isolated in the USA by Albert Sabin from a 6-year-old boy who died of toxoplasmic encephalitis in 1939 [1]. This RH strain is highly virulent in mice causing $100 \%$ lethality, irrespective of the dose. Since 1939, this RH strain has been maintained in mice or in cell culture in many laboratories worldwide and has become the reference T. gondii strain. Almost all of what we currently know about cell biology, immunology and genetics of this organism has been discovered from the RH strain. In 1948, Sabin and Feldman used the RH strain to develop the dye-test which is still the 'gold standard' for the serological diagnosis of toxoplasmosis.

Since the isolation of the RH strain in 1939, several laboratories involved in the diagnosis of human toxoplasmosis in the USA and France have isolated other clinical strains in mice. These strains were first isolated from severe cases of congenital toxoplasmosis and later, with the expansion of the AIDS epidemics, from cases of toxoplasmic encephalitis. Some strains exhibited an $\mathrm{RH}$-like high virulence in mice, but very infrequently. In fact, the vast majority of clinical strains were avirulent in mice. Genetic variability among strains was the most likely explanation for these different patterns of virulence observed in mice. Strain genotyping was initiated in the early 1990s by David Sibley and John Boothroyd in the USA and by Marie-Laure Dardé in France. With different genetic markers, their results were highly similar: more than $95 \%$ of strains belonged to one of three major clonal lineages known as types I, II and III $[2,3]$. The RH strain and the other highly virulent strains for mice were classified into lineage I, whereas the avirulent strains were clustered into lineages II or III.
Further studies revealed a much more complicated population structure of $T$. gondii. In fact, early genotyping studies were conducted where strains were available, that is in the USA and France from clinical samples for diagnosis procedures and from domestic animal samples such as sheep and pigs collected in abattoirs. There was a major sampling bias because strains from wildlife and from other parts of the world were largely under-represented in the genetic analysis. In the early 2000s, thanks to the great work of Dubey and collaborators, numerous strains have been collected from chickens in Central and South America, especially in Brazil. Later, the analysis with multilocus markers of these strains showed an abundance of atypical genotypes that did not fit the conventional classification of three major lineages described in the USA and France [4]. Now we wait for data from Africa and Asia, where $75 \%$ of the current world human population is living, to unravel the secrets of the population structure of this fascinating and successful parasite that is currently infecting one-third of the world's population.

The big question is: does the genotype of T. gondii influence the severity of toxoplasmosis?

The high genetic diversity of Toxoplasma strains in the tropical zone of the Americas may partly explain some clinical characteristics observed in this area. The prevalence of ocular toxoplasmosis is highest in Brazil, especially in the southern city of Erechim where up to $17.7 \%$ of 1042 adults were found to have retinal scars clinically related to toxoplasmosis [5]. In the Guianas, highly divergent strains have been collected in otherwise healthy adults who acquired life-threatening disseminated toxoplasmosis after consumption of wild game or contaminated water in the Amazon rainforest [6].

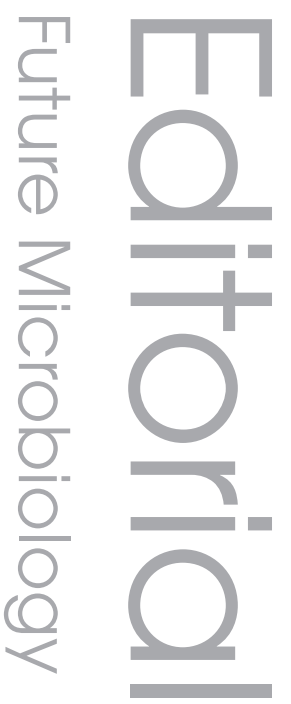

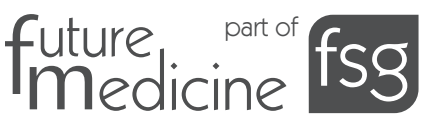


"In immunocompromised persons, type II strains are predominant, regardless of the underlying cause of immunosuppression, site of infection or outcome."
The 'Amazonian toxoplasmosis' and the high rate of ocular toxoplasmosis in southern Brazil are likely attributable to the genetic makeup of atypical strains circulating in these areas, even though other factors such as high inoculum exposure or genetic background of the host may be involved.

What about human toxoplasmosis in Europe and the USA where three clonal lineages predominate? A literature-based answer to this question is that type I strains are associated with acquired ocular toxoplasmosis, congenital toxoplasmosis and cerebral toxoplasmosis in immunocompromised persons [7]. Based on my experience within the Toxoplasma Biological Resource Center (Toxo BRC), I disagree with this overused statement. Toxo BRC is a French network of parasitologists in metropolitan France and in French overseas departments of French West Indies and French Guiana. Since 2002, this network is organized for the systematic collection of Toxoplasma isolates from patients with toxoplasmosis, together with clinical and epidemiological data of these patients. From 2002 to date, I have successfully performed a multilocus microsatellite analysis of T. gondii isolates from more than 500 patients who presented with a wide spectrum of clinical manifestations and I have never identified a type I strain (i.e., an RH-like strain) in these patients. Type II strains are overwhelmingly involved in congenital toxoplasmosis in France whatever the clinical presentation, in asymptomatic or subclinical cases and in severe neuro-ophthalmic involvement or fetal death in utero [8]. In immunocompromised persons, type II strains are predominant, regardless of the underlying cause of immunosuppression, site of infection or outcome [9]. However, the genotype of Toxoplasma strains is strongly linked to the presumed geographical origin of infection: immunocompromised patients commonly reactivate a type II strain if acquired in Europe and an atypical strain if acquired in sub-Saharan African countries, French overseas departments or South America.

Why, then, is it so generally believed that type I strains are associated with human toxoplasmosis in Europe and the USA? In my opinion, the importance of type I strains in toxoplasmosis has been largely overestimated in the literature owing to a collection bias, or 'iceberg effect', and method issues.

Type I strains isolated in mice from clinical samples are from rare historical cases and are over-represented in genotyping studies. Early genetic analyses of $T$. gondii were based on a collection of strains accumulated in two reference centers, one in the USA and the other in France, during the period 1939-1994. The abundance of strains in a collection of a reference center depends on what colleagues send to this center. As underlined before, in the USA and in France, avirulent strains are the rule; virulent strains are the exception that proves the rule. The strain collection of both reference centers was like the visible part of an iceberg; type I strains were over-represented because they had killed the mice, which is unusual. Owing to their rarity, these unusual strains were sent to the reference centers. The part of an iceberg below the water, or mouse nonvirulent type II strains, were rarely sent to the reference centers because they were basic strains. Of note, in France, no type I strain has been isolated in mice since 1994.

Since 1997, the genotyping analysis of most studies has been performed directly from DNA extracted from clinical samples without isolation of strains in mice. Since T. gondii is scarce in human samples, a nested PCR assay has been used in order to increase the sensitivity of the PCR assay. A big concern with nested PCR is that it is extremely easy to get contamination even in strictly controlled environments. The $\mathrm{RH}$ strain produces millions of tachyzoites in cell culture or in mouse ascitic fluid and is at very high risk of contaminating the nested PCR products when it is used as a positive control.

In 1997, it was advocated using only one marker (SAG2) for genotyping $T$. gondii strains, since it is capable of distinguishing all three clonal lineages at a single locus. This turned out to be a mistake [10]. If a Toxoplasma DNA carries one allele characteristic of type I strains, it does not mean that all alleles are identical to those of type I strains. We have to be cautious even when using three or four markers because, if we look at additional markers, different polymorphisms may be revealed 'transforming' a 'type I strain' into a nontype I strain, as I have experienced myself and as have others [10,11]. Anyway, the results of studies identifying type I strains by monolocus typing are of no value unless they are confirmed by additional multilocus typing or evidenced by a high virulence pattern in mice.

Type I strains in human toxoplasmosis: myth or reality? In my opinion, it is a myth. It is fascinating, however, to see how this myth, based on studies largely opened to criticism, spread in the literature to finally be widely accepted by the scientific community. 


\section{Financial \& competing interests disclosure}

The author has no relevant affiliations or financial involvement with any organization or entity with a financial interest in or financial conflict with the subject matter or materials discussed in the manuscript.
This includes employment, consultancies, honoraria, stock ownership or options, expert testimony, grants or patents received or pending, or royalties.

No writing assistance was utilized in the production of this manuscript.

\section{Bibliography}

1. Sabin AB: Toxoplasmic encephalitis in children. J. Am. Med. Assoc. 116, 801-807 (1941).

2. Howe DK, Sibley LD: Toxoplasma gondii comprises three clonal lineages: correlation of parasite genotype with human disease. J. Infect. Dis. 172, 1561-1566 (1995).

3. Dardé ML: Biodiversity in Toxoplasma gondii. Curr. Top. Microbiol. Immunol. 219, 27-41 (1996).

4. Lehmann T, Marcet PL, Graham DH, Dahl ER, Dubey JP: Globalization and the population structure of Toxoplasma gondii. Proc. Natl Acad. Sci. USA 103, 11423-11428 (2006).
5. Glasner PD, Silveira C, Kruszon-Moran D et al.: An unusually high prevalence of ocular toxoplasmosis in southern Brazil. Am. J. Ophthalmol. 114, 136-144 (1992).

6. Carme B, Demar M, Ajzenberg D, Dardé ML: Severe acquired toxoplasmosis caused by wild cycle of Toxoplasma gondii, French Guiana. Emerg. Infect. Dis. 15, 656-658 (2009).

7. Pereira-Chioccola VL, Vidal JE, Su C: Toxoplasma gondii infection and cerebral toxoplasmosis in HIV-infected patients. Future Microbiol. 4, 1363-1379 (2009).

8. Ajzenberg D, Cogné N, Paris L et al.: Genotype of 86 Toxoplasma gondii isolates associated with human congenital toxoplasmosis, and correlation with clinical findings. J. Infect. Dis. 186, 684-689 (2002).
9. Ajzenberg D, Yera H, Marty P et al.: Genotype of 88 Toxoplasma gondii isolates associated with toxoplasmosis in immunocompromised patients and correlation with clinical findings. J. Infect. Dis. 199, 1155-1167 (2009).

10. Khan A, Jordan C, Muccioli C et al:: Genetic divergence of Toxoplasma gondii strains associated with ocular toxoplasmosis, Brazil. Emerg. Infect. Dis. 12, 942-949 (2006).

11. Khan A, Su C, German M, Storch GA, Clifford DB, Sibley LD: Genotyping of Toxoplasma gondii strains from immunocompromised patients reveals high prevalence of type I strains. J. Clin. Microbiol. 43, 5881-5887 (2005). 